Vol tammet ri c di screte cur rent of pol yani I i ne- coated I at ex particles at mi cr oel ect r odes

\begin{tabular}{|l|l|}
\hline 著者 & AOK Koi chi, Ke Qi ang \\
\hline $\begin{array}{l}\text { j our nal or } \\
\text { publ i cat i on } \mathrm{titl} \text { e }\end{array}$ & Journal of El ectroanal yt i cal Chemi stry \\
\hline vol une & 587 \\
\hline number & 1 \\
\hline page range & $86-92$ \\
\hline year & $2006-02$ \\
\hline URL & ht t p: //hdl . handl e. net $/ 10098 / 1773$ \\
\hline
\end{tabular}




\title{
Voltammetric discrete current of polyaniline-coated latex particles at microelectrodes
}

\author{
Koichi Aoki*, Qiang Ke \\ Department of Applied Physics, University of Fukui, 3-9-1 Bunkyo, Fukui-shi, 910-8507 \\ Japan, d930099@icpc00.icpc.fukui-u.ac.jp
}

\begin{abstract}
Suspensions of polyaniline-coated latex particles $13 \mu \mathrm{m}$ in diameter showed the integer-fold voltammetric currents at the microelectrode of which size was similar to the diameter of the particles. The currents did not vary with concentrations of polyaniline particles but varied accidentally with each voltammetric run. They were caused by the reduction of a few polyaniline particles adsorbed on the electrode rather than diffusion of polyaniline particles in the suspension. The voltammetric behavior was almost the same as at the large electrode except for the integer-fold currents. The particle was a nearly mono-dispersed sphere, and was composed of a polystyrene core and a polyaniline shell. The particles were dispersed in acidic aqueous solution to form a colloidal suspension. The redox charge per particle was $11 \mathrm{G}$ redox electrons, one-third of which participated in the voltammetric reduction current.
\end{abstract}

key words: polyaniline, latex particles, core-shell, colloidal suspensions, microelectrodes 


\section{Introduction}

Detection of individual events of reactions is intriguing in that the events bring out an image of a dynamic path of reacting molecules. It has been carried out by means of fluorescence techniques [1-5] overcoming difficulty in sensitivity as well as rapidity of the response. The fluorescence techniques have been applied even to detection of electrochemical individual events [6]. The electrochemical information of the individual event might desirably involve charge transfer and mass transport, as have been observed in conventional current-potential curves. In order to monitor electrochemically the individual events, current should be amplified, discriminated against a noise level. Fan and Bard trapped an ion in a microscopic space, and detected the current $[7,8]$ with the help of amplification due to the redox cycle [9]. Another method of the amplification is to use redox particles with a number of charges such as redox dendrimers [10,11], redox latex particles [12-25] and nanoparticles of metals [26-31]. The big redox particles are expected to reveal a macroscopic view including mass transport, collision with an electrode, and a charge transfer step at the collision.

This concept has led us to synthesize such large redox particles as polystyrene latexes immobilizing hydrogen ion [12-17], polyaniline [18-21] and ferrocenyl moiety [22-25]. Since some of the redox particles can be seen through an optical microscope, the electrode behavior has been observed simultaneously together with positioning the particles by the microscope [16]. With an increase in size of particles, complications may occur in size-distribution, aggregation in suspension, adsorption on an electrode [22] and partial charge transfer [23-25]. The first three problems have been able to be solved by selecting empirically synthetic conditions, whereas the last includes an essential subject relevant to charge propagation within a particle.

A question associated with electrode reactions of huge particles arises: what behavior is observed at an electrode of which size is close to the particles. This question has been conceived during the study on nanometer-sized electrodes in the context of effects of 
diffuse-double layer [32-34] and apparently large values of charge transfer reaction rates [35-39]. However, it is still under debate mainly because of insufficiently reproducible data about voltammograms and electrode geometry to analyze them quantitatively. This viewpoint has allowed us to answer the above question by carrying out voltammetry of micrometer-sized redox latex particles at a micrometer-sized electrode. Several types of latex of conducting polymers have been synthesized [40-43] to yield mono-dispersed particles. In this report, we show concentration-independent integer-fold voltammetric currents of suspensions of polyaniline-coated latex particles $13 \mu \mathrm{m}$ in diameter at the microelectrode similar to the 10 $\mu \mathrm{m}$ in diameter. This behavior is ascribed to the random adsorption of a few redox particles on the electrode.

\section{Experimental Section}

\section{Chemicals.}

Styrene (Wako) was purified by distillation under vacuum at $60{ }^{\circ} \mathrm{C}$ and stored in a refrigerator until use. Poly( $N$-vinyl pyrrolidone) (PVP)(Wako) of $360 \mathrm{~kg} \mathrm{~mol}^{-1}$ was used as a surfactant during the dispersion polymerization of styrene. 2,2'-Azoisobutyronitrile (AIBN) (Kanto) was used as an initiator in the preparation of seed of latexes. Ethanol (99.5 \%, Wako), 2-methoxyethanol (Wako), ammonium peroxydisulfate (Kanto), aniline hydrochloride (Kanto), and 2-propanol were used as received. All aqueous solutions were prepared with deionized water.

\section{Preparation of particles.}

Seed particles were prepared by the dispersion polymerization of styrene in the mixed solvent of $54 \mathrm{~cm}^{3}$ ethanol and $36 \mathrm{~cm}^{3}$ methoxyethanol including $1.5 \mathrm{~g}$ PVP in a three-necked round-bottomed flask. $15 \mathrm{~cm}^{3}$ Styrene was added to the mixture, in which $0.33 \mathrm{~g}$ AIBN, an initiator of the polymerization, was dissolved. The polymerization was performed in nitrogen 
atmosphere during mechanical stirring at $70^{\circ} \mathrm{C}$ for $24 \mathrm{~h}$. The produced latex was rinsed extensively with distilled water by applying centrifugation-dispersion process several times. The product was used as seed for the synthesis of big polystyrene particles.

$100 \mathrm{~cm}^{3}$ Suspension of the $10 \%(\mathrm{v} / \mathrm{v})$ seed was dispersed in the mixture of $54 \mathrm{~cm}^{3}$ ethanol, $36 \mathrm{~cm}^{3}$ methoxyethanol, $1.5 \mathrm{~g}$ PVP, $15 \mathrm{~cm}^{3}$ styrene and $0.33 \mathrm{~g}$ AIBN. Conditions of the polymerization and the purification were the same as those performed for the seed. The polymerization and the purification were iterated three times. The purified latex was used for the synthesis of polyaniline-coated polystyrene latex.

After $8.6 \mathrm{mmol}$ aniline hydrochloride was added to $100 \mathrm{~cm}^{3}$ of $4 \%$ suspension of the big latex, the mixture was stirred in an ice bath for $2 \mathrm{~h}$ in order to adsorb aniline on the surface of the latex. When the oxidant, $2.45 \mathrm{~g}$ ammonium persulfate, was added to the mixture, the polymerization was performed at $0{ }^{\circ} \mathrm{C}$ for $5 \mathrm{~h}$. The mixture was stirred at room temperature for a further $14 \mathrm{~h}$ to complete the polymerization. The suspension was centrifuged by means of a refrigerated centrifuge, SRX-201(TOMY, Tokyo) to prevent degradation of polyaniline ${ }^{6 e}$. The centrifugation separated the mixture of the product into three layers. The second layer contained fragment of polyaniline. The bottom layer, which contained a high proportion of the polyaniline-coated latex, was redispersed in $0.1 \mathrm{M}$ sulfuric acid, and then centrifuged. The re-dispersion and centrifugation were iterated several times until the top layer became clear.

\section{Electrochemistry and microscopy.}

Platinum disks, $1.6 \mathrm{~mm}$ and $0.01 \mathrm{~mm}$ in diameter, were purchased (BAS, Tokyo) for a voltammetric working electrode. The electrode surface was polished with $0.05 \mu \mathrm{m}$ alumina powder before each experiment. The platinum wire and the $\mathrm{Ag} \mid \mathrm{AgCl}$ electrode were used as a counter electrode and a reference electrode, respectively. Voltammetric measurements were made using a HECS-1112 potentiostat (Fuso, Kawasaki) in latex suspensions including $1 \mathrm{M}$ $\mathrm{H}_{2} \mathrm{SO}_{4}$ and PVP.

A digital optical photomicrograph of the latex particles was obtained with a VMS-1900 (Scalar) video microscope and was transferred to a PC using a MPG capture board. Scanning 
electron microscopy (SEM) studies were carried out using a S2400 (Hitachi).

\section{Results and Discussion}

\subsection{General features of latex.}

Since polyaniline is hydrophilic, the polymerization of aniline may occur only on the surface of polystyrene latex. Thus the synthesized latex should be composed of the polystyrene-core which is covered with the polyaniline layer [21]. The polyaniline-coated polystyrene latex (PANI-PS) particles, showing dark green, were almost spherical. The average diameter was $2 a=13.03 \pm 0.12 \mu \mathrm{m}$ evaluated from SEM while the diameter from the optical microscope was $2 a=12.7 \pm 0.3 \mu \mathrm{m}$, where the errors denote the standard deviation. The larger value by the SEM than by the optical microscope may be ascribed to the difference in circumstances of the samples; Pt-coating in vacuum for the SEM and the aqueous suspension for the optical microscope.

Although the polystyrene-core was synthesized by growing iteratively, it is nearly mono-dispersed, as is shown in Fig. 1. The diffusion coefficient was estimated from the Stokes-Einstein equation [44] to have $D=0.375 \times 10^{-9} \mathrm{~cm}^{2} \mathrm{~s}^{-1}$ at $25^{\circ} \mathrm{C}$ in water with viscosity of $0.891 \mathrm{~g} \mathrm{~m}^{-1} \mathrm{~s}^{-1}$. The PANI-PS particles were dispersed in aqueous solution to yield a stable suspension with at least 1 year's life even if PVP was not included in the suspension. Observation by the video microscope showed that each particle was subject to Brownian motion. The supernatant obtained by centrifugation was transparent, and hence polyaniline is confined to the latex without discharging any detectable fragment of polyaniline in the suspension.

Polystyrene is dissolved well in tetrahydrofuran (THF) whereas polyaniline cannot be dissolved in THF. We immersed the dried PANI-PS latex of $52.7 \mathrm{mg}$ in THF for 1 day in order to dissolve the core, and weighed the dried remnant, the PANI shell, to obtain $8.3 \mathrm{mg}$. Letting the radii of the latex and of the core be $a$ and $b$, respectively, the weight ratio of the latex to the shell is given by 
$\left\lfloor b^{3} d_{\mathrm{S}}+\left(a^{3}-b^{3}\right) d_{\mathrm{A}}\right\rfloor /\left\lfloor\left(a^{3}-b^{3}\right) d_{\mathrm{A}}\right\rfloor=52.7 / 8.3$

where $d_{\mathrm{S}}$ and $d_{\mathrm{A}}$ are densities of polystyrene $\left(1.05 \mathrm{~g} \mathrm{~cm}^{-3}\right)$ and of polyaniline $\left(1.46 \mathrm{~g} \mathrm{~cm}^{-3}\right)$ [45], respectively. Then we obtain $a / b=1.04$ or $b=6.26 \mu \mathrm{m}$ for $a=6.52 \mu \mathrm{m}$. The thickness of polyaniline layer $(a-b)$ is $0.26 \mu \mathrm{m}$. The average density and the mean mass of the particle are $1.10 \mathrm{~g} \mathrm{~cm}^{-3}$ and $1.27 \mathrm{ng}$. The amount of polyaniline per particle is $0.20 \mathrm{ng}$. In order to evaluate the redox charge per particle, we obtained a relationship between the redox charge and weight of an electrochemically polymerized polyaniline film. The weight, $0.47 \mathrm{mg}$, was obtained by the difference in the weights of a Pt wire before and after polymerization of aniline. The charge was obtained by integration of the voltammogram in $1 \mathrm{M} \mathrm{H}_{2} \mathrm{SO}_{4}$ at several scan rates $\left(1-20 \mathrm{mV} \mathrm{s}^{-1}\right)$. The ratio of the redox charge to the weight was $7.6 \mathrm{C} \mathrm{g}^{-1}$. Consequently, one PANI-PS particle has $1.5 \mathrm{nC}$, corresponding to $0.95 \times 10^{10}$ electrons for charge transfer reaction per particle.

\subsection{Voltammetry at large electrode}

Figure 2 shows the cyclic voltammogram of the PANI-PS suspension. The cathodic wave was observed at ca. $0.05 \mathrm{~V}$ vs. $\mathrm{Ag} \mid \mathrm{AgCl}$ in the suspension, whereas a very small and broad anodic wave appeared at ca. $0.25 \mathrm{~V}$. These waves were discriminated against the voltammogram of the supernatant, which was close to the voltammogram of the blank solution. Therefore they are not due to polyaniline fragments broken and scatted in the solution, but due directly to the PANI-PS particles. Polyaniline of the PANI-PS after the synthesis should take the emeraldine form or the conducting state because it was generated with the oxidation polymerization. Indeed, no wave appeared in the potential domain from 0.3 to $0.9 \mathrm{~V}$ when the potential was scanned from $0.2 \mathrm{~V}$. The wave at $0.05 \mathrm{~V}$ can be attribute to the redox conversion of emeraldine to leucoemeraldine (the insulating state). The wave at the second scan was smaller than the first one because of a loss of the anodic wave. The small current of the anodic wave contrasts with the sharp anodic peak the electropolymerized polyaniline film at $0.2 \mathrm{~V}$, as is shown in the inset of Fig. 2. The irreversible reaction has been 
explained [21] in terms of an insufficiently short period of the conducting particle with an electrode for propagation time of the conducting zone [46-48].

The previous PANI-PS particles ca. $1 \mu \mathrm{m}$ in diameter showed diffusion-controlled peak current [21]. The cathodic peak current, $I_{\mathrm{p}}$, for the present PANI-PS suspension was plotted against the square-root of the scan rate, $v$, in Fig. 3, revealing the proportionality. According to the theory for linear sweep voltammetry of multi-charged particles, the diffusion-controlled peak current density is expressed by [49]

$j_{\mathrm{p}}=0.446 n F c *(v D F / R T)^{1 / 2}$

A point of this equation is a loss of $n^{1 / 2}$, which is ascribed to the combination of the Nernst equation and the diffusion equation. The slope calculated from Eq.(1) for known $n, c^{*}$ and $D$, was drawn in Fig. 3 as a dashed line. The experimental value is only $10 \%$ of the theoretical diffusion-controlled current. The smaller value can be ascribed either to the partial charge transfer owing to a delay of charge propagation $[24,25]^{19}$ or inappropriate application to diffusion of the latex suspension. The latter will be supported in the next section.

The background-subtracted voltammogram had sometimes a cathodic peak and sometimes a plateau of a limiting current. We plotted the cathodic peak current or the limiting current against the concentration of the PANI-PS in Fig. 4, expecting a proportional relation. However, the peak current did not vary with the concentration, implying that the diffusion model is in vain. When the water was sprayed onto the electrode surface which was taken out from the suspension, some particles were adsorbed on the surface against the water spray, as is shown in Fig. 5. Therefore, the cathodic current is caused by the reduction of the adsorbed PANI-PS. The invariance of the current to the concentration suggests saturation of the adsorption of the PANI-PS. If the PANI-PS particles are adsorbed in a hexagonally close-packed mono-particle layer with $91 \%$ fractional packing, the number of the particles on the electrode (area is $A=0.02 \mathrm{~cm}^{2}$ ) is $1.26 \times 10^{4}\left(=0.91 A / \pi a^{2}\right)$. Then, the redox charge on the electrode is $\Gamma=1.26 \times 10^{4} n e=1.92 \times 10^{-5} \mathrm{C}$ for $n=0.95 \times 10^{-9}$. If the charge transfer reaction at one redox site is Nernstian with one-electron step and if there is no mass transport in the adsorbed layer, the voltammetric peak current of the adsorbed layer is expressed by 
$I_{\mathrm{p}}=\Gamma v(F / 4 R T)$

This value is $5.6 \mu \mathrm{A}$ for $v=0.03 \mathrm{~V} \mathrm{~s}^{-1}$, being the one-third of the experimental value. The voltammetric peak current of adsorbed species should exhibit the proportionality to $v$ rather than $v^{1 / 2}$. This contradiction from Fig. 3 can be explained in terms of the diffusion-like charge transfer process, which has often been observed in thick polyaniline films [50-53].

It is now necessary to interpret the meaning of $n, c^{*}$ and $D$ in Eq.(1) from the viewpoint of mass transport of the redox moiety in polyaniline rather than the mass transport of PANI-PS particles. We regard $n c^{*}$ and $D$ as the concentration of the redox site of polyaniline and the apparent diffusion coefficient for the charge exchange. This model is equivalent to the diffusion-like charge transport in a film on the electrode. If the mono-particle layer of PANI-PS were to be uniform in the distribution of the redox site, the concentration of the redox site, $n c^{*}$, $(n=1)$ could be estimated from the redox charge, $1.92 \times 10^{-5} \mathrm{C}$, of the electrode divided by $2 a A F$ to yield $7.7 \mathrm{mM}$. Inserting this value in Eq.(1) for the slope in Fig.3 $\left(I_{\mathrm{p}} v^{-1 / 2}=9.0 \mu \mathrm{A} \mathrm{V} \mathrm{V}^{-1 / 2} \mathrm{~s}^{1 / 2}\right)$ gives a value of the apparent diffusion coefficient, $D_{\mathrm{ap}}=$ $5 \times 10^{-8} \mathrm{~cm}^{2} \mathrm{~s}^{-1}$. This value is reasonable for the apparent diffusion coefficient for the charge exchange. Therefore the reduction current is modeled as a charge exchange reaction in the thin film which is composed of the mono-particle layer of PANI-PS.

\subsection{Voltammetry at microelectrode}

Figure 6 shows a cyclic voltammogram in the PANI-PS suspension at the microelectrode $10 \mu \mathrm{m}$ in diameter. The waveform at the microelectrode was actually the same as at the large electrode, with showing the hysteresis current. Therefore it is not ascribed to spherical diffusion characteristic to the microelectrode behavior but to the surface process. The surface process can be seen from the decrease in the cathodic current with an increase in the number of the scan. The ratio of the current at the large electrode to that at the microelectrode is $10^{4}$, whereas the ratio of the electrode area is $2.6 \times 10^{4}$. The similarity of the order of the ratios supports the surface process. We evaluated the total charge of the background-subtracted 
voltammogram from the integration of the current-potential curve in the potential domain from 0.4 to $0.01 \mathrm{~V}$. The charge was $0.47 \mathrm{nC}$, which is $1 / 3$ of the redox charge $(1.5 \mathrm{nC})$ of one particle of the polyaniline. The front of the reduced zone may reach the one-third of the adsorbed particle at the scan rate, as is illustrated in Fig. 6.

We varied concentration of the PANI-PA suspension, expecting the independence of the concentration, similar to in Fig. 5. However, the variation was in chaos, as in shown in Fig. 7, although each voltammetry was performed immediately after polishing the electrode surface with alumina powder. Since this microelectrode exhibited the quite reproducible diffusion-controlled steady-state current for the oxidation of ferrocenylmethyltrimethyl ammonium hexafluorophosphate in aqueous solution, it has no trouble in structure. A careful stare of the chaotic variation allows us to notice the localization of the data points close to 0 , 0.03 and $0.06 \mathrm{nA}$. We attempted to sort the current values in the ascending order, and denoted the $m$-th sorted current as $I_{m}$. In other words, $m$ means the number of the current points less than $I_{m}$. Figure 8 (circles) shows variation of $m$ with the current, $I_{m}$. If the current values were to be distributed randomly and uniformly, the plot of $m$ against the current should fall on a straight line all over the data with common separation between two closest neighboring points. However, the plot shows not only a concave variation but also some groups. In order to stress the variation, we differentiated the variation numerically, according to the finite difference between closest neighboring points

$\frac{\mathrm{d} m}{\mathrm{~d} I} \approx \frac{\Delta m}{\Delta I}=\frac{1}{I_{m}-I_{m-1}}$

Figure 8 shows the finite difference values (triangles) computed from Eq.(3). The data points are assembled to some groups, 0, 25-40, 52-64 and 81-96 pA. These groups increase stepwise each by 31 pA. Consequently, the current is digitized. The number of the occurrence, $L$, included in each group is shown as the height of a bar in Fig. 8. The first digitized current occurred preferentially.

From the sizes of the disk electrode and the particles, we can image the geometry of the particles and electrode as illustrated in Fig. 9. Since the diameter of the particle is a little 
larger than the disk diameter, only one particle can come in contact with the electrode surface (Fig. 9(A)). A second and a third particle can have electrochemical contact with the electrode via the directly contacting particle, as is illustrated in Fig. 9(B) and (C). These images can be supported with the photographs of one (A in Fig. 10) and two or three (B,C in Fig. 10) adsorbed PANI-PS particles on the Pt electrode in the suspension. When the potential was switched between $-0.05 \mathrm{~V}$ and $0.50 \mathrm{~V}$, the particles exhibited color change, demonstrating the electrode reaction. The current of the adsorbed particles should be independent of the area of the electrode but vary with the number, $k$, of the particles directly and the indirectly contacting with the electrode. Replacing $c^{*}$ in Eq.(2) by the $\Gamma / 2 a$, we obtain the expression of the peak current as

$I_{\mathrm{p}}=0.446 k(\Gamma / 2 a)\left(v D_{\mathrm{ap}} F / R T\right)^{1 / 2}$

The value of $I_{\mathrm{p}} / k$ for $\Gamma=0.47 \mathrm{nC}, D_{\text {ap }}=5 \times 10^{-8} \mathrm{~cm}^{2} \mathrm{~s}^{-1}$ and $v=0.03 \mathrm{~V} \mathrm{~s}^{-1}$ is $39 \mathrm{pA}$, which is close to the quantitized current, 31 pA for Fig. 8.

The frequency, $L$, in Fig. 8 decreases with an increase in $k$. Taking the ratio of $L$ at $k$ to $k$-1, we obtain $L_{k=2} / L_{k=1}=0.4, L_{k=3} / L_{k=2}=0.5$ and $L_{k=4} / L_{k=3}=0.4$. The similarity of these values indicates that a group of $k$ particles on the electrode is generated from the group of $k-1$ particles with the common probability, 0.4-0.5. The common probability may be ascribed to transport of a particle in the suspension to the group of $k$ particles and then to adhesion to the group. Since the probability is close to the ratio of the radius of the particle to the average distance, $d$, between neighboring particles, ranging 0.36 to 0.66 (Fig. 8) for the concentration range, $0.05-0.32 \mathrm{~g} \mathrm{~cm}^{-3}$ (Fig. 7), the particle may attach randomly to the group to increase $k$.

\section{Conclusion}

The discrete voltammetric current of the PANI-PS suspension was observed at the microelectrode of which size was smaller than the redox latex particles. The discreteness is caused by the integer number of the particles in electric contact with the electrode. The reduction current was controlled by diffusion-like charge exchange at the redox sites within 
the adsorbed PANI-PS rather than physical diffusion of the particles in the suspension. Since the voltammetric electrolysis time is shorter than the duration of the whole charge exchange, the voltammetrically discrete charge was one-third of the whole charge per particle. The discreteness is not caused by the property of the electric conduction but is ascribed to the redox property. Consequently, it is a general feature of electrode reaction at an electrode of which size is close to the size of redox particles. Since the discreteness occurs by random attachment of a particle in the suspension, it is not controllable.

\section{Acknowledgement}

This work was financially supported by Grants-in-Aid for Scientific Research (Grants 16550070 and 14340232) from the Ministry of Education in Japan.

\section{Reference}

[1] W. M. Itano, J. C. Bergquist, D. J. Wineland, Science 237 (1987) 612.

[2] H. Dehmelt, W. Paul, N. F. Ramsey, Rev. Mod. Phys. 62 (1990) 525.

[3] E. Betzig, J. K. Trautman, T. D. Harris, J. S. Weiner, R. L. Kostelak, Science 251 (1991) 1468.

[4] X. S. Xie, R. C. Dunn, Science 265 (1994) 361.

[5] W. P. Ambrose, P. M. Goodwin, J. C. Martin, R. A. Keller, Phys. Rev. Lett. B 72 (1994) 160.

[6] M. M. Collinson, R. M. Wightman, Science 268 (1995) 1883.

[7] F. R. F. Fan, A. J. Bard, Science 267 (1995) 871.

[8] A. J. Bard, F. R. F. Fan, Acc. Chem. Res. 29 (1996) 572.

[9] K. Aoki, M. Morita, O. Niwa, H. Tabei, J. Electroanal. Chem. 256 (1988) 269.

[10] C. Amatore, Y. Bouret, E. Maisonhaute, J. I. Goldsmith, H. D. Abruna, Chemphyschem. 2 (2001) 130.

[11] D. J. Diaz, G. D. Storrier, S. Bernhard, K. Takada, H. D. Abruna, Langmuir 15 (1999) 7351.

[12] J. M. Roberts, P. Linse, J. G. Osteryoung, Langmuir 14 (1998) 204.

[13] K. Aoki, J. M. Roberts, J. G. Osteryoung, Langmuir 14 (1998) 4445. 
[14] J. M. Roberts, J. J. O'Dea, J. G. Osteryoung, Anal. Chem. 70 (1998) 3667.

[15] K. Aoki, T. Lei, Electrochem. Commun. 1 (1999) 101.

[16] K. Aoki, C. Wang, Langmuir 17 (2001) 7371.

[17] K. Aoki, C. Wang, J. Chen, J. Electroanal. Chem. 540 (2003) 135.

[18] T. Lei, K. Aoki, J. Electroanal. Chem. 482 (2000) 149.

[19] T. Lei, K. Aoki, K. Fujita, Electrochem. Comm. 2 (2000) 290.

[20] K. Aoki, T. Lei, Langmuir 16 (2000) 10069.

[21] K. Aoki, J. Chen, Q. Ke, S. P. Armes, D. P. Randall, Langmuir 19 (2003) 5511.

[22] J. Chen, C. Xu, K. Aoki, J. Electroanal. Chem. 546 (2003) 79.

[23] C. Xu, J. Chen, K. Aoki, Electrochem. Comm. 5 (2003) 506.

[24] C. Xu, K. Aoki, Langmuir 20 (2004) 10194.

[25] J. Chen, Z. J. Zhang, J. Electroanal. Chem. (2005) in press.

[26] K. Aoki, J. Chen, N. Yang, H. Nagasawa, Langmuir 19 (2003) 9904.

[27] N. Yang, K. Aoki, H. Nagasawa, J. Phys. Chem. B 108 (2004) 15027.

[28] N. Yang, K. Aoki, Electrochim Acta, 50 (2005) 4868.

[29] J. F. Hicks, D. T. Miles, R. M. Murray, J. Am. Chem. Soc. 124 (2002) 13322.

[30] K. H. Ng, H. Liu, R. M. Penner, Langmuir 16 (2000) 4016.

[31] J. Liu, L.Cheng, Y. Song, B. Liu, S. Dong, Langmuir 17 (2001) 6747.

[32] J. L. Conyers Jr., H. S. White, Anal. Chem., 72 (2000) 4441.

[33] V. P. Menon, C. R. Martin, Anal. Chem. 67 (1995) 1920.

[34] J. D. Norton, H. S. White, S. W. Feldberg, J. Phys. Chem. 94 (1990) 6772.

[35] J. Chen, K. Aoki, Electrochem. Commun. 4 (2002) 24.

[36] K. Aoki, Electrochem. Commun. 7 (2005) 523.

[37] R. M. Penner, M. J. Heben, T. L. Longin, N. S. Lewis, Science 250 (1990) 1118.

[38] M. V. Mirkin, T. C. Richards, A. J. Bard, J. Phys. Chem., 97 (1993) 7672.

[39] J. J. Watkins, J. Chen, H. S. White, H. D. Abruna, E. Maisonhaute, C. Amatore, Anal. Chem. 75 (2003) 3962.

[40] D.K. Moon, K. Osakada, T. Maruyama, T. Yamamoto, Makromol. Chem., 193 (1992) 829.

[41] P. Beadle, S. P. Armes, S. Gottesfeld, C. Mombourquette, R. Houlton, W. D. Andrews, S. F. Agnew, Macromolecules, 25 (1992) 2526.

[42] C-F. Liu, T. Maruyama, T. Yamamoto, Polym. J., 25 (1993) 363.

[43] A. E. Wiersma, L. M. A. vd Steeg, T. J. M. Jongeling, Synth. Met., 71 (1995) 2269.

[44] P. W. Atkins, Physical Chemistry, Sixth edition, Oxford University Press, Oxford, 1998, 
p.749.

[45] C. Barthet, S. P. Armes, S. F. Lascelles, S. Y. Luk, H. M. E. Stanley, Langmuir 14 (1998) 2032.

[46] Y. Tezuka, S. Ohyama, T. Ishii, K. Aoki, Bull. Chem. Soc. Jpn. 64 (1991) 2045.

[47] K. Aoki, T. Aramoto, Y. Hoshino, J. Electroanal. Chem. 340(1992) 127.

[48] T. F. Otero, I. Boyano, M. T. Cortés, G. Vázquez, Electrochim. Acta 49 (2004) 3719.

[49] K. Aoki, Electroanalysis 17 (2005) 1379.

[50] E. M. Genies, C. Tsintavis, J. Electroanal. Chem. 200 (1986) 127.

[51] M. Kalaji, L. Nyholm, L. M. Peter, J. Electroanal. Chem. 313 (1991) 271.

[52] M. Grzeszczuk, G. Zabinska-Olszak, J. Electroanal. Chem. 359 (1993) 161.

[53] V. F. Ivanov, O. L. Gribkova, A. A. Nekrasov, A. V. Vannikov, J. Electroanal. Chem. 372 (1994) 57. 


\section{Figure Captions}

Fig. 1. SEM photograph of dried PANI-PS.

Fig. 2. Cyclic voltammograms of (solid curve) $0.074 \mathrm{~g} \mathrm{~cm}^{-3}$ PANI-PS suspension in $1 \mathrm{M}$ $\mathrm{HCl}+1.5 \mathrm{wt} \%$ of PVP and (dotted curve) $1 \mathrm{M} \mathrm{HCl}+1.5 \mathrm{wt} \%$ of PVP at the scan rate of $0.05 \mathrm{~V} \mathrm{~s}^{-1}$. The inset is cyclic the voltammogram of electrochemically synthesized polyaniline on the Pt electrode.

Fig. 3. Dependence of the cathodic peak current at $0.05 \mathrm{~V}$ on the square-root of the scan rate in the $0.074 \mathrm{~g} \mathrm{~cm}^{-3}$ PANI-PS suspension. The dashed line was calculated from Eq.(1) for known values of $n, c^{*}, D$ and the area of the electrode $\left(2.00 \mathrm{~mm}^{2}\right)$.

Fig. 4. Dependence of the cathodic peak current at $0.05 \mathrm{~V}$ on concentrations of the PANI-PS suspension containing $1 \mathrm{M} \mathrm{H}_{2} \mathrm{SO}_{4}$ at $v=0.03 \mathrm{~V} \mathrm{~s}^{-1}$.

Fig. 5. Photograph of the surface of the Pt electrode observed with the optical microscope after the one-cycle of voltammetric measurement.

Fig. 6. Multiple-scanned voltammogram of the $0.102 \mathrm{~g} \mathrm{~cm}^{-3}$ PANI-PS suspension containing $1 \mathrm{M} \mathrm{H}_{2} \mathrm{SO}_{4}$ without PVP at $v=0.03 \mathrm{~V} \mathrm{~s}^{-1}$ at the Pt electrode $10 \mu \mathrm{m}$ in diameter. The inset is an illustration of charge exchange of polyaniline from the contacting point of a PANI-PS with the electrode.

Fig. 7 Dependence of the cathodic peak current at $0.1 \mathrm{~V}$ on concentrations of PANI-PS suspensions containing $1 \mathrm{M} \mathrm{H}_{2} \mathrm{SO}_{4}$ at $v=0.03 \mathrm{~V} \mathrm{~s}^{-1}$ at the Pt electrode $10 \mu \mathrm{m}$ in diameter.

Fig. 8. Plot of (circles) the event number, $m$, for the current less than $I_{m}$, and the plot of (triangles) the finite difference of the variation of $m$ vs. , i.e., $\left(I_{m}-I_{m-1}\right)^{-1}$ against $I_{m}$. A bar graph denotes the frequency, $L$, occurring at each discreet domain. 
Fig. 9. Illustrations of (A) one, (B) two and (C) three adsorbed particles on the microelectrode

Fig. 10. Photographs of (A) one PANI-PS particle on the Pt electrode $20 \mu \mathrm{m}$ in diameter, (B) two or three particles on the Pt electrode $10 \mu \mathrm{m}$ in diameter, and (C) side view of the two particles on the glass-covered Pt electrode $30 \mu \mathrm{m}$ in diameter. 


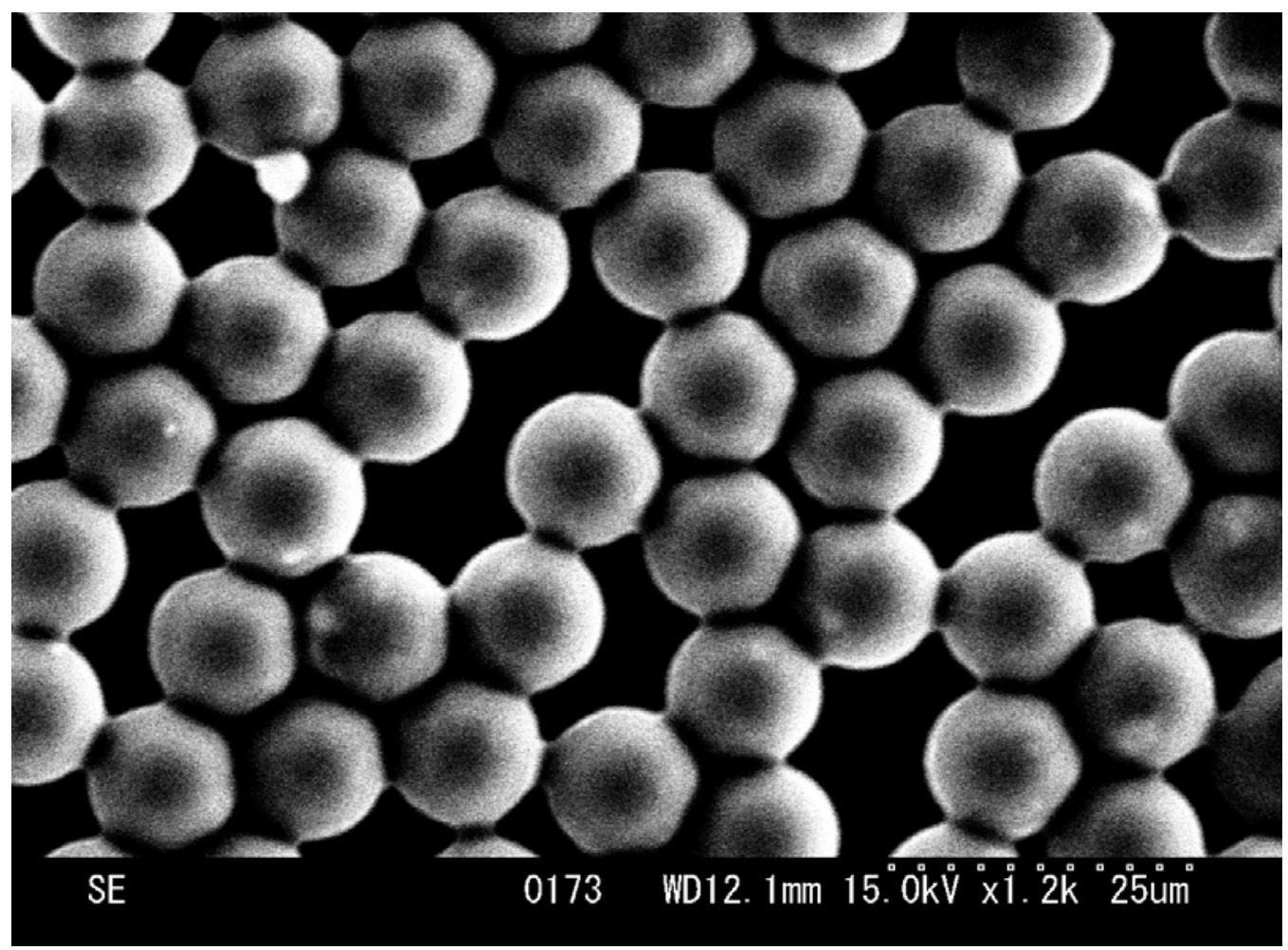

Figure 1

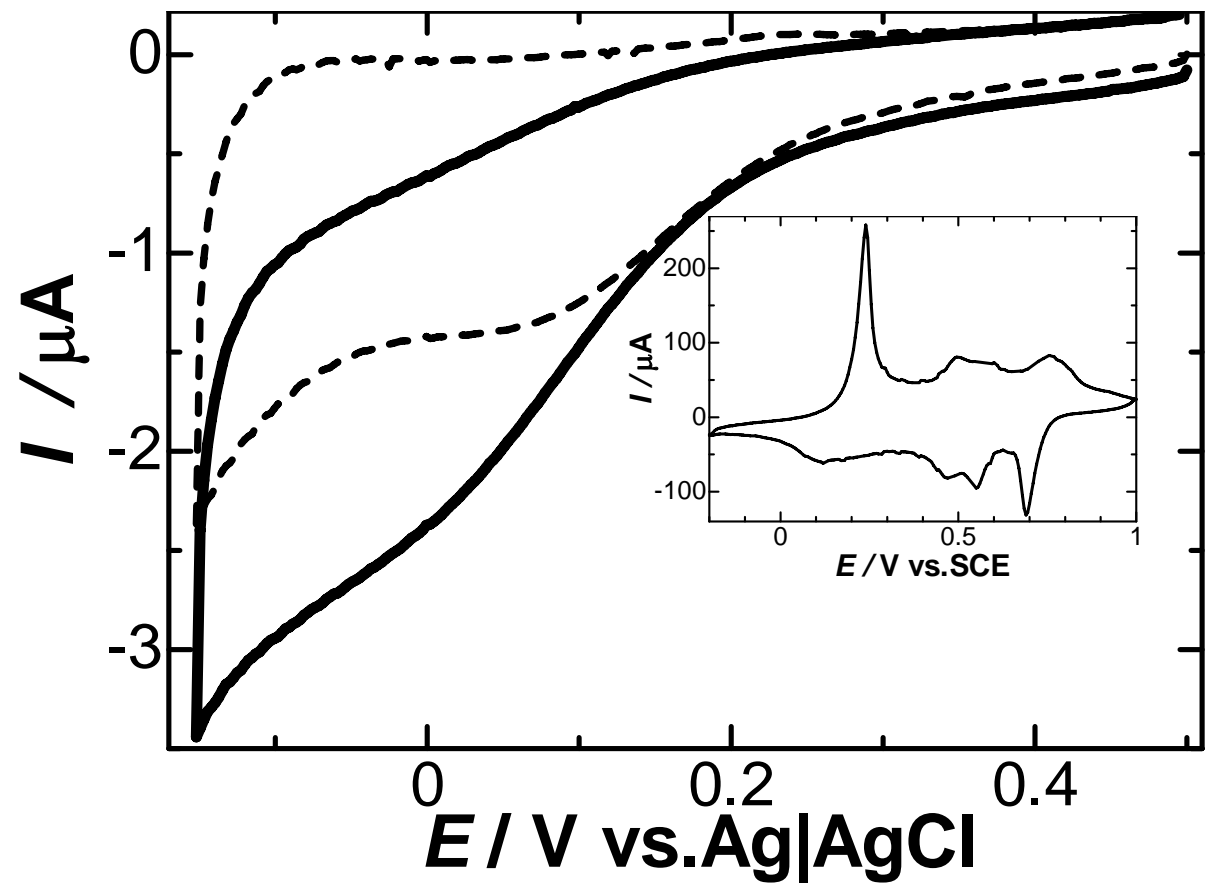

Figure 2 


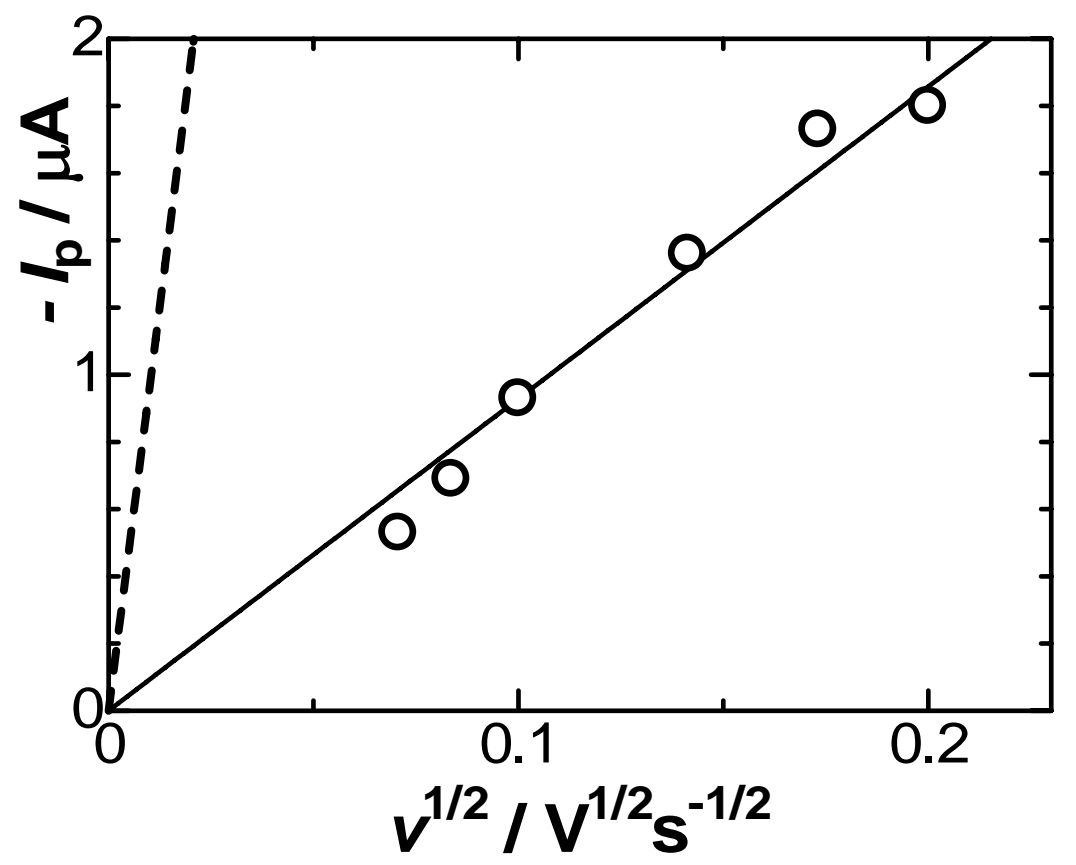

Figure 3

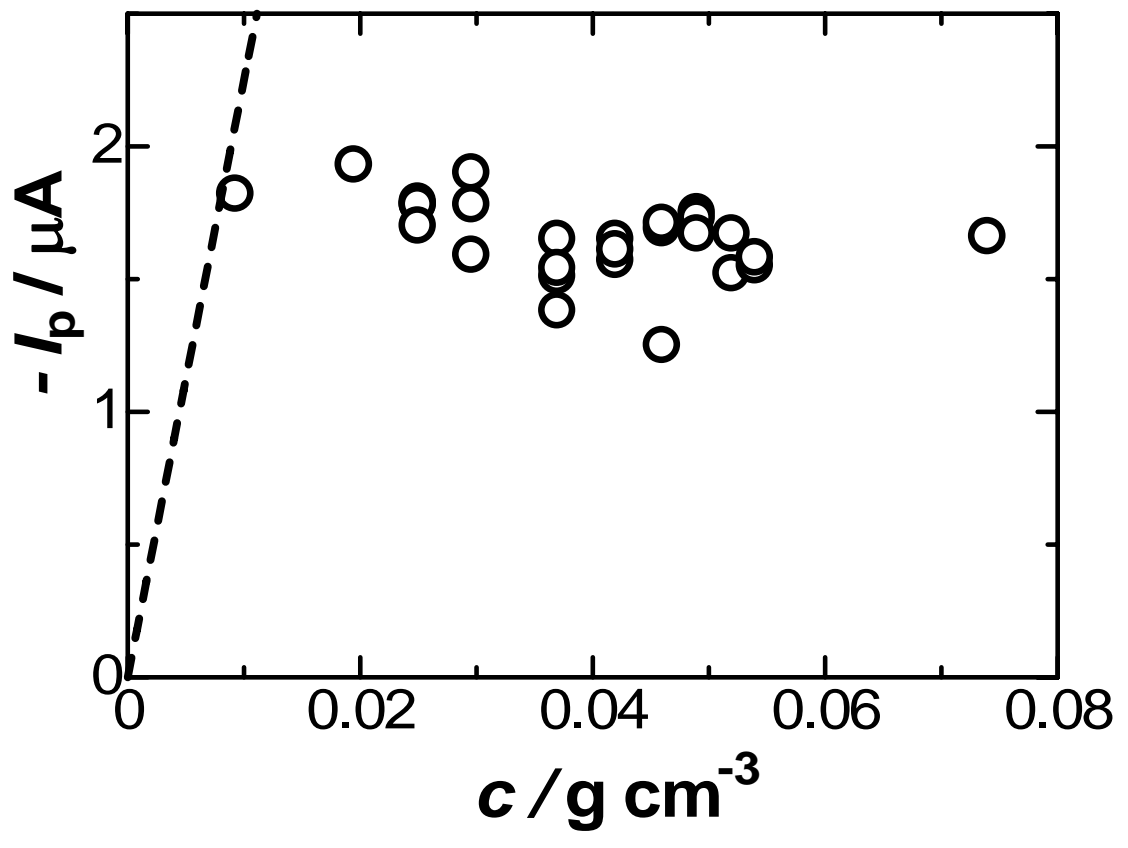

Figure 4 


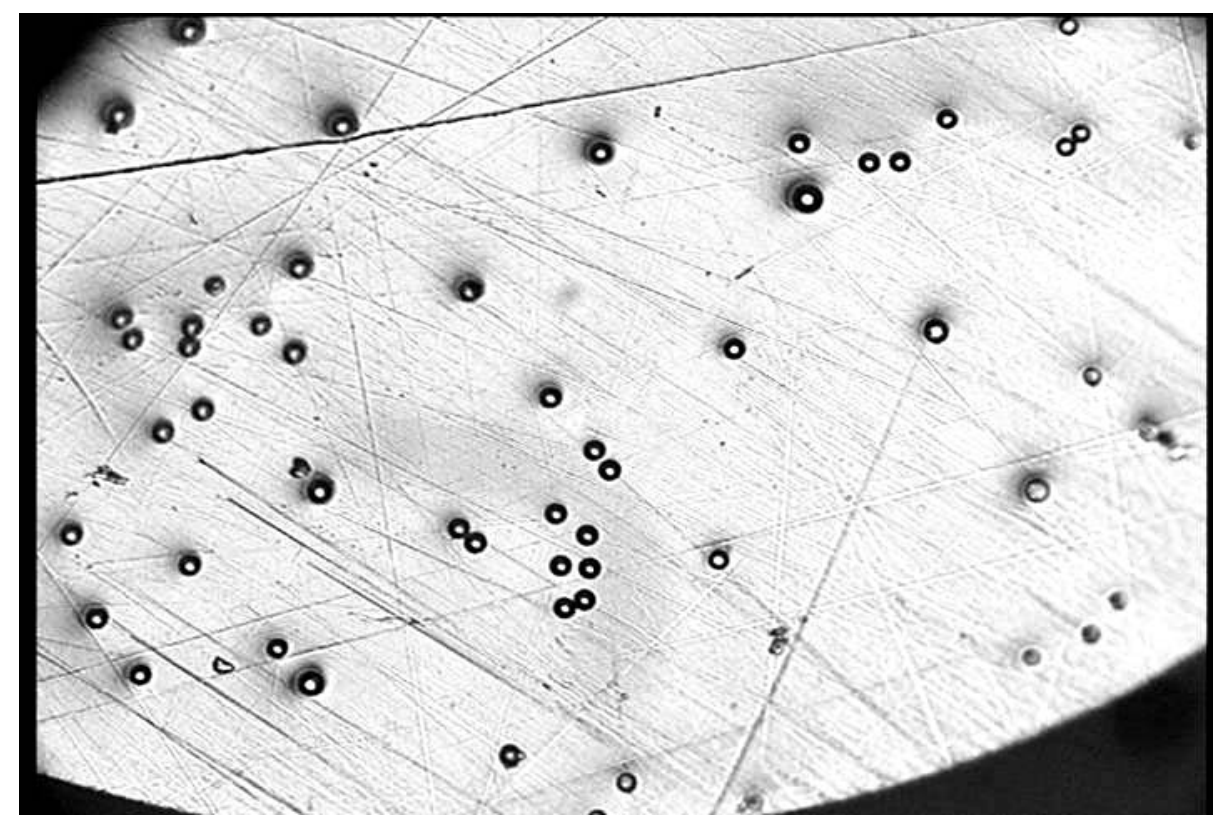

Figure 5

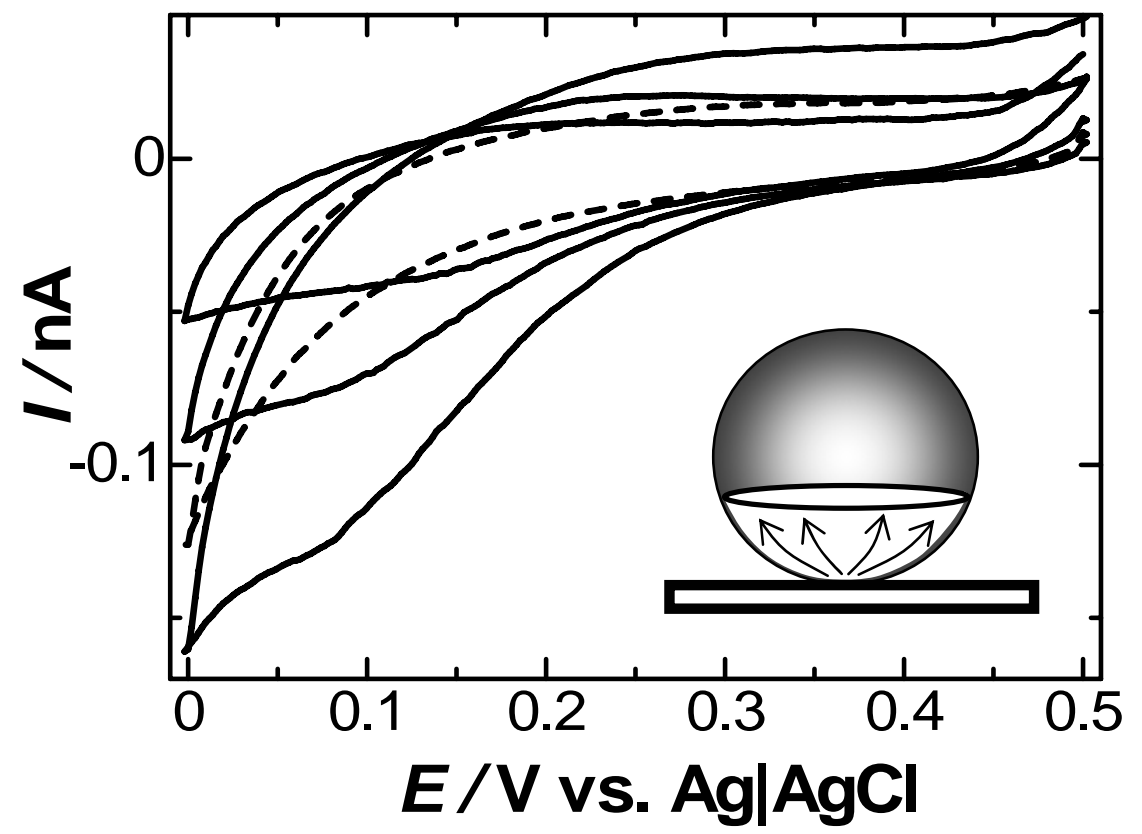

Figure 6 


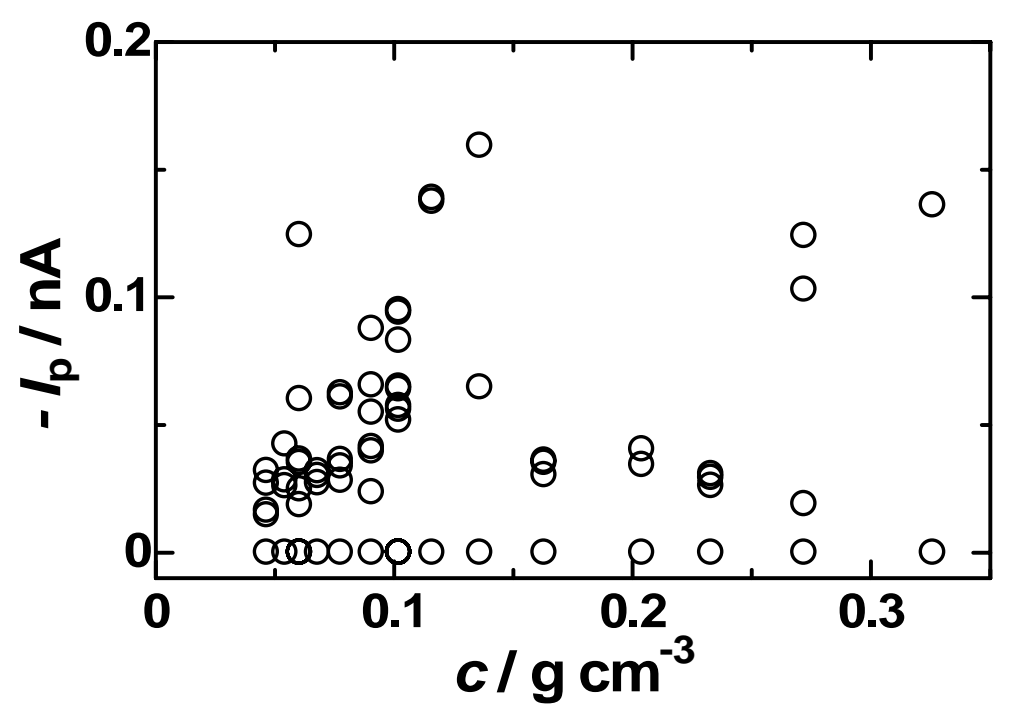

Figure 7

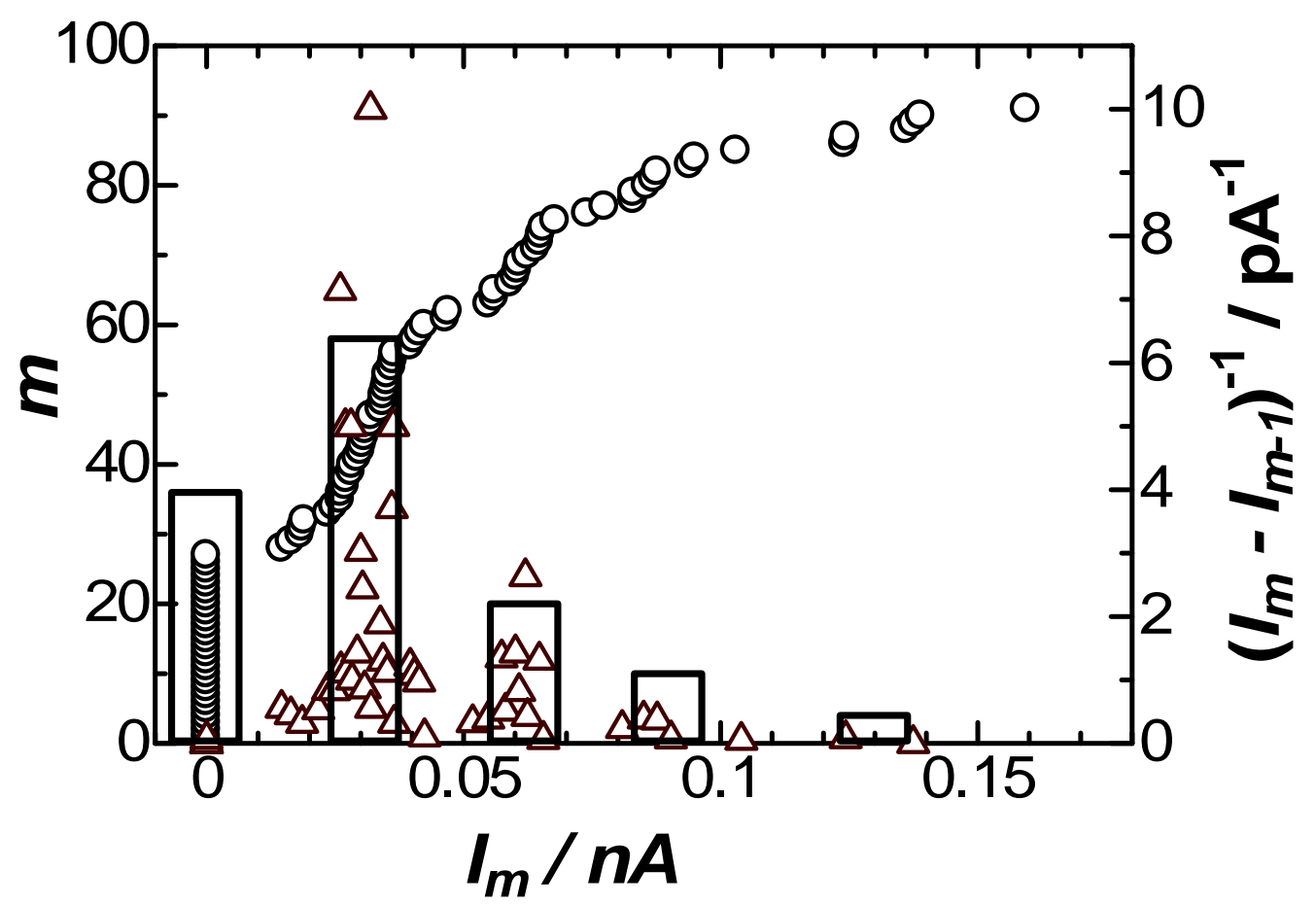

Figure 8 


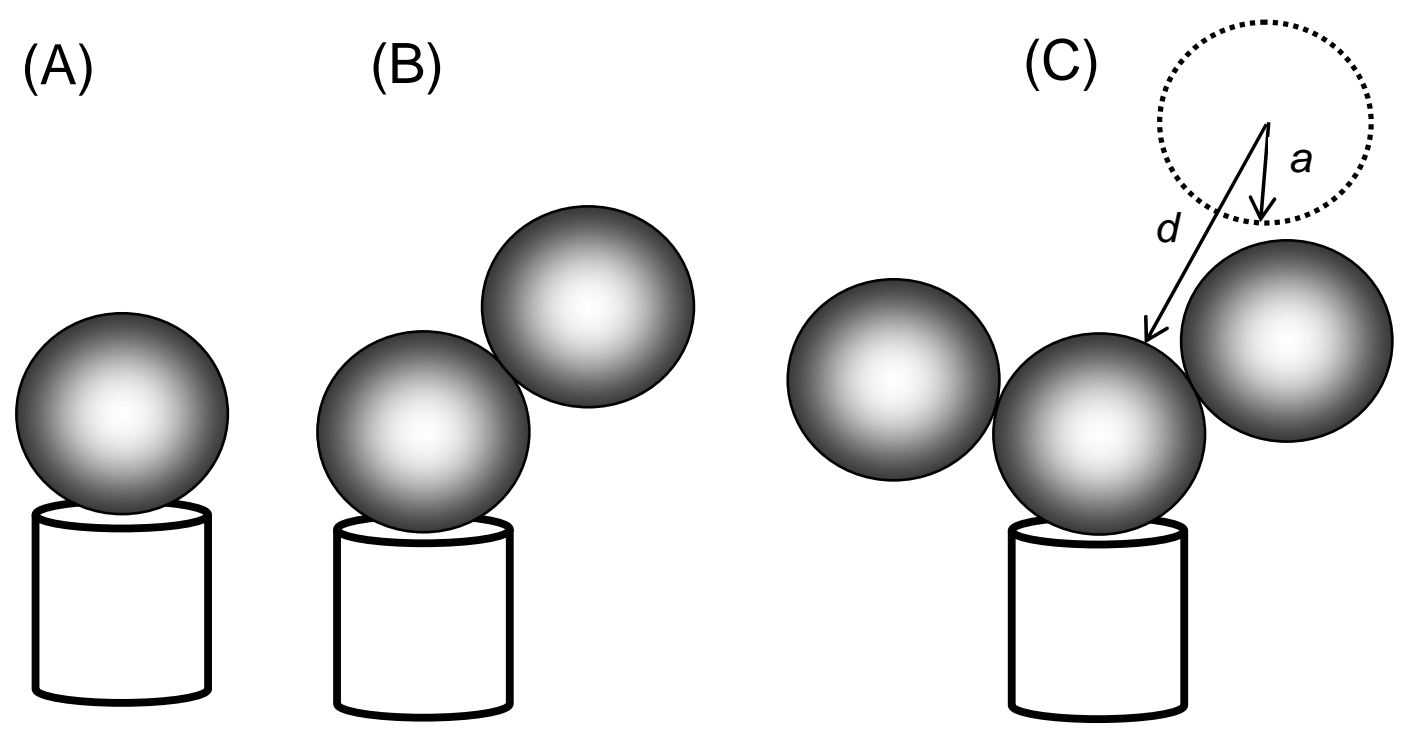

Figure 9

(A)

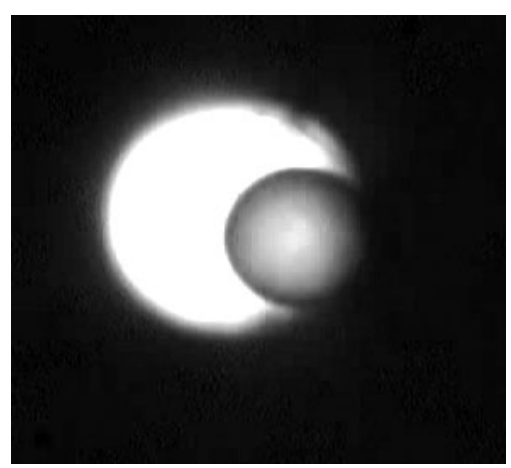

(B)

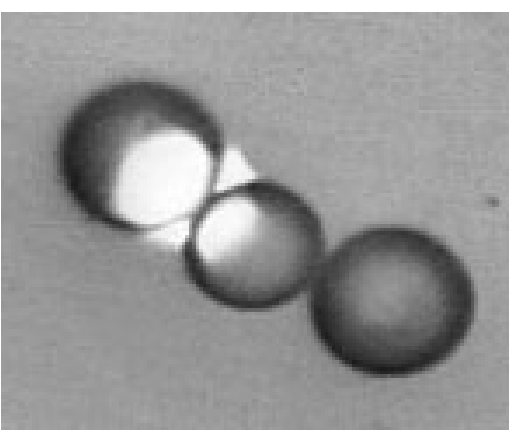

(C)

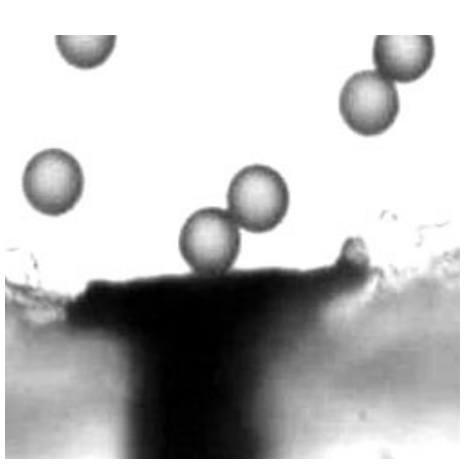

Figure 10 